\title{
Theory of consolidation and precursory time of earthquakes
}

\author{
Naoto Kaneko ${ }^{1}$ and Hiroyuki Nagahama ${ }^{1}$ \\ ${ }^{l}$ (Department of Earth Science, Graduate School of Science, Tohoku University, Japan)
}

\begin{abstract}
Settlement of ground can be explained by Terzaghi's theory of consolidation in the field of soil mechanics. Terzaghi introduced the concept of strain to the consolidation equation in the theory. On the other hand, the diffusion-like equation for earthquake prediction can explain precursory phenomena such as crustal movement and electrical resistivity closely link to the relationship between magnitude and precursor time. However, the reason is obscure why derived from the diffusion-like equation in spite of given seismological impact. Accordingly, we noticed the void ratio from the point of view of consolidation rather than the equation by the concept of hydrostatic pressure. Therefore, this consolidation idea (to relate void ratio and dilatancy) can effectively explain the electrical resistivity composed in saturated ground.
\end{abstract}

Keywords: Consolidation, Dilatancy, Earthquake, Porous medium equation, Precursory time

\section{Introduction}

Liquefaction by earthquake sometimes can induce severely damage to our life. When geo-deformation phenomena caused by consolidation settlement are described, we conventionally classified the field of earthquake and consolidation independently. Because of different fields, we study seismology or geology for the earthquake and soil mechanics or civil engineering for consolidation. In this study, we would like to receive the baton from both fields and previous research for the goal of earthquake prediction. Firstly, the earthquake prediction in light of dilatancy model has been proposed actively in 1970's. Water diffusion in rocks could explain a large class of phenomena precursory to earthquakes such as crustal movement, electrical resistivity, radon emission, seismic shear velocity anomaly and $b$ value of Gutenberg-Richter law [1]. These observed data were fitted as a coordinate axis of magnitude and precursor interval (lead time). However it is heuristics and obscure solid foundation for the diffusion-like equation. In addition half a century ago, Terzaghi's theory of consolidation has been instrumental in bearing capacities of footings of structure and its analysis in spite of onedimension [2]. Here, we indicate essence on saturated ground settlement process links to above.

\section{Critical state soil mechanics}

Critical state [3] at ground (including soil and rock) can be derived from flow rule and some constitutive laws [4] as follows:

Flow rule is used by plastic potential $f$ is given by [5];

$d \dot{\varepsilon}^{p}=h \frac{\partial f}{\partial \sigma_{i j}^{\prime}}, h>0$,

where $d \dot{\varepsilon}^{p}$ is incremental tensor of plastic strain rate, $\sigma_{i j}^{\prime}$ is effective stress tensor, and $h$ is plastic multiplier. Increment of plastic strain tensor is partial differential of the potential due to effective stress in $q-p^{\prime}-v$ space, where $q$ is the deviatoric stress, $v$ is the specific volume and $p^{\prime}$ is the mean effective stress. When stress state of soil reaches to the critical state [3], the state of soil yields and behaves fluidity. Regarding plastic potential on $q-$ $p$ plane, it causes plastic expansion on the upper side of the critical state line and causes plastic compression on the lower side of the line. Therefore, the critical state line can be understood as condition boundary line. Then, the slope of critical state line is expressed by $M$ can be defined by

$q=M p^{\prime}$.

On the other hand, the work increment at shear process has been assumed to equal the energy dissipation at the critical state $[6,7]$.

$p^{\prime} d \varepsilon_{v}+q d \varepsilon_{d}=M_{c} p^{\prime} d \varepsilon_{d}$,

where $\varepsilon_{v}$ is volumetric strain and $\varepsilon_{d}$ is deviatoric strain. From Eq. (3), we can obtain

$\frac{q}{p^{\prime}}=M_{c}-\left(\frac{d \varepsilon_{v}}{d \varepsilon_{d}}\right)$,

Moreover, Rowe [8] microscopically studied Eq. (4) from the view point of deformation of granular materials and obtained a similar relation as 


$$
\frac{\sigma_{1}}{\sigma_{3}}=K\left(\frac{-d \varepsilon_{3}}{d \varepsilon_{1}}\right)=-\frac{2 \dot{\varepsilon}_{3}}{\dot{\varepsilon}_{1}} \tan ^{2}\left(\frac{\pi}{4}+\frac{\varphi}{2}\right),
$$

where $\sigma_{1}$ is major principal stress, $\sigma_{3}$ is minor principal stress, $\varepsilon_{1}$ is axial strain and $\varepsilon_{3}$ is lateral strain. This is an expression for the relationship between principal stress ratio $\left(=\sigma_{1} / \sigma_{3}\right)$ and principal strain incremental ratio during the shear of granular materials $\left(=-d \varepsilon_{3} / d \varepsilon_{1}\right)$ and is usually called stress-dilatancy relation. Moreover, $K$ is internal energy ratio of soil. Besides, we can regard Eq. (2) or Eq. (4) as Eq. (5). Then, the outward plastic strain increment vector components $\left(d \varepsilon_{v}^{p}\right.$ and $\left.d \varepsilon_{d}^{p}\right)$ orthogonally intersects as shown by Eq. (1). At this point, normal vector of plastic potential plane is equivalent to the yield function of $\dot{\varepsilon}^{p}$ on $q-p^{\prime}$ plane. Then, we obtain

$$
\frac{d \varepsilon_{v}^{p}}{d \varepsilon_{d}^{p}}=-\frac{d q}{d p^{p}} .
$$

From Eqs. (4) and (6), we can get

$$
\frac{q}{p^{\prime}}-\frac{d q}{d p^{\prime}}=M_{c} \text {. }
$$

Then, setting from the case $\left(p^{\prime}=p_{y}, q=0 ; p_{y}\right.$ is a hardening parameter) and integrating Eq. (7), the following relation is obtained as

$f=\frac{q}{M_{c} p^{\prime}}+\ln \left(\frac{p^{\prime}}{p_{0}^{\prime}}\right)-\ln \left(\frac{p_{y}}{p_{0}^{\prime}}\right)$,

where $F$ is called the yield function of Cam-clay model. By using $p_{y}$, the plastic void ratio $e^{p}$ is given by [7],

$d e^{p}=-(\lambda-\kappa) \frac{d p_{y}}{p_{y}}$,

where $\kappa$ is swelling index and $\lambda$ is compression index at the critical state line. On the other hand, the relationship between plastic volumetric strain $\varepsilon_{v}^{p}$ and plastic volume ratio $v^{p}$ is given by [9],

$$
d \varepsilon_{v}^{p}=\frac{d v^{p}}{v}=-\frac{d e^{p}}{1+e},
$$

where $v$ is expressed by void ratio $e(v=1+e)$.

\section{Terzaghi's consolidation theory}

The amount of settlement of ground is expressed by the well-known consolidation model $[2,10]$,

$$
e=e_{0}-C_{\mathrm{c}} \log \frac{\sigma^{\prime}}{\sigma_{0}^{\prime}},
$$

where $e_{0}$ is initial void ratio, $C_{c}$ is compression index, and $\sigma^{\prime}$ is effective vertical stress (especially $\sigma_{0}{ }^{\prime}$ is initial effective vertical stress). Moreover, Eq. (11) is proposed by Terzaghi $[2,10]$ who fitted experimental consolidation data. The slope of consolidation line, which is $\sigma^{\prime}$ more increases as the means of $e$ decreases, the equation is obtained,

$$
-\frac{d e}{d \sigma^{\prime}}=m_{v}\left(1+e_{0}\right),
$$

where $m_{\mathrm{v}}$ is the coefficient of volume compressibility, and $\left(1+e_{0}\right)$ expresses the previous volume of soil. From Eq. (12), we can get

$$
-\frac{\delta e}{1+e_{0}}=m_{v} \delta \sigma^{\prime}=\frac{\delta S_{t}}{\delta z},
$$

where $z$ is the total thickness of layer, and $S_{t}$ is the amount of settlement. Moreover, Eq. (13) can significantly link to Eq. (10). Then, we introduce the infinitesimal time of change to Eq. (13) as follows;

$\frac{\partial v_{a}}{\partial z}=\frac{-1}{1+e_{0}} \frac{\partial e}{\partial t}=m_{v} \frac{\partial \sigma^{\prime}}{\partial t}$,

where $t$ is the time, and $v_{a}$ is the displacement rate which is taken as positive downwards in the direction of $z$. On the other hand, total stress is constant, so pore-pressure $u$ is given by

$$
\frac{\partial \sigma^{\prime}}{\partial t} \equiv-\frac{\partial u}{\partial t} \text {. }
$$

From Eqs. (14) and (15), we can get

$$
\frac{\partial v_{a}}{\partial z}=-m_{v} \frac{\partial u}{\partial t} \text {. }
$$


By the Darcy's law, the relationship between $v_{a}$ and $u$ is given by,

$v_{a}=k i=k\left(-\frac{\partial h_{l}}{\partial z}\right)=-\frac{k}{\gamma_{w}} \frac{\partial u}{\partial z}$

where $i$ is hydraulic gradient, $h_{l}$ is loss of head and $\gamma_{\mathrm{w}}$ is density of water. From Eqs. (16) and (17), we can get

$\frac{k}{\gamma_{w}} \frac{\partial^{2} u}{\partial z^{2}}=-\frac{\partial v_{a}}{\partial z}=m_{v} \frac{\partial u}{\partial t}$

Thus

$\frac{\partial u}{\partial t}=c_{v} \frac{\partial^{2} u}{\partial z^{2}}$

where $c_{\mathrm{v}}=k /\left(\gamma_{\mathrm{w}} m_{\mathrm{v}}\right)$ is the coefficient of consolidation. Eq. (19) is called Terzaghi's fundamental differential equation for one-dimensional consolidation formula $[9,10]$, and can be derived based on the concept of critical state.

\section{Precursory time of earthquakes}

We introduced in view of the consolidation the ground composed of saturated, so we could predict the amount and time of settlement. Relatedly, the diffusion-like equation was given as

$\frac{\partial P}{\partial t}=c \nabla^{2} P$,

where $P$ is the fluid pressure, and $c$ is hydraulic diffusivity (constant value) [1]. Then, Eqs. (19) and (20) are clearly connected each. Eq. (20) attentively describes flow in a porous medium. Moreover, the data of precursory phenomena of earthquakes [1] are fit to $\tau=L^{2} / c$, where $\tau$ is precursor time in days, and $L$ is the longest dimension of the aftershock zone of the earthquake. On the other hand, the relation [11] between magnitude $\left(M_{L}\right)$ and $\tau$ from observational data as

$\log \tau=0.80 M_{L}-1.92$.

Additionally, the relationship between the magnitude and the fault length of earthquakes is given by [12, see also 13],

$M_{L}=(1 / 0.6) \log L+4.85$.

Putting all these evidences together, $\tau$ is approximately proportional to $L^{2}$ [11], and $M_{L}$ can be converted to $L$. However, there is much uncertainty in all of observed parameters [11]. Precursor time $\tau$ depends on hydraulic diffusivity. Eq. (13) can express the deformation of ground as stain. Therefore, the volumetric strain $e$ is a suitable measurement for the dilatancy before earthquake, and electrical resistivity which depends on void ratio and water contain. The rate of change dilatancy temporally can be analyzed directly by Eq. (19).

\section{Conclusion}

In this study, we discuss the earthquake precursor time based on the Terzaghi's consolidation equation which is equivalent to diffusion-like equation on precursory phenomena for earthquakes.

\section{References}

[1] Scholz, C. H., Sykes, L. R., and Yash, P. A., Earthquake Prediction: A Physical Basis, Science, 181(4102), 1973, 803-810.

[2] Terzaghi, K., Erdbaumechanik auf bodenphysikalischer Grundlage (Leipzig u. Wien: F. Deuticke), 1925, 399p.

[3] Schofield, A., and Wroth, P., Critical State Soil Mechanics (London: McGRAW-HILL), 1968, 310p.

[4] Kaneko, N., Muto, J. and Nagahama, H., Concept of plasticity index to influence mechanical bifurcation of soils and soft-sediment deformation, Second International Conference on Science, Engineering \& Environment (SEE2016), held in Osaka International House Foundation, Osaka city, Japan, Nov.21-23, 2016, in press.

[5] Mises, R. von., Mechanik der plastischen Formänderung von Kristallen, Z. angew., Math. Mech. 8(3), 1928, 161-185

[6] Roscoe, K. H., Schofield, A. N. and Wroth, C. P., On the yielding of soils, Géotechnique, 8(1), 1958, $22-53$.

[7] Roscoe, K. H., Schofield, A. N. and Thurairajah, A., Yielding of clays in state wetter than critical, Géotechnique, 13(3), 1963, 211240 .

[8] Rowe, P. W., The stress-dilatancy relation for static equilibrium of an assembly of particles in contact, Proc. Roy. Soc. Lond. A, 269(1339), 1962, 500-527.

[9] Butterfield, R., A natural compression law for soils (an advance on $e$-log p'), Géotechnique, 29(4), 1979, 469-480.

[10] Terzaghi, K., and Peck, R. B., Soil Mechanics in Engineering Practice (New York: JOHN WILEY \& SONS), 1948, 566p.

[11] Whitcomb, J. H., Garmany, J. D., and Anderson, D. L., Earthquake prediction: Variation of seismic velocities before the San Francisco earthquake, Science, 180(4086), 1973, 632-635.

[12] Matsuda, T., Magnitude and recurrence interval of earthquake from a fault, Zisin (J. Seismol. Soc. Jpn.) 2nd ser., 28(3), 1975, 269283. (in Japanese with English abstract)

[13] Nagahama, H., Self-affine growth pattern of earthquake rupture zones, Pure and Appl. Geophys., 142(2), 1994, $263-271$. 